\title{
Nordiques
}

\section{Adventures in a Borderless Land: Myth, Modernity and the Environment in Máret Ánne Sara's In Between Worlds (2014)}

Silje Solheim Karlsen

\section{CpenEdition}

\section{Journals}

Electronic version

URL: http://journals.openedition.org/nordiques/446

DOI: $10.4000 /$ nordiques.446

ISSN: 2777-8479

Publisher:

Association Norden, Bibliothèque de Caen la mer

\section{Printed version}

Date of publication: 1 May 2019

Number of pages: $95-130$

ISBN: 979-1-0959140-3-7

ISSN: 1761-7677

Electronic reference

Silje Solheim Karlsen, "Adventures in a Borderless Land: Myth, Modernity and the Environment in Máret Ánne Sara's In Between Worlds (2014)", Nordiques [Online], 37 | 2019, Online since 31 October 2020, connection on 14 March 2021. URL: http://journals.openedition.org/nordiques/446 ; DOI: https:// doi.org/10.4000/nordiques.446 


\section{Une nature arctique qui protège et nourrit : le contre-récit des Kvènes récrit la relation au monde naturel}

Anitta Viinikka-Kallinen*

\section{RÉSUMÉ}

Les Kvènes, une minorité nationale de la Norvège, ont longtemps été assujettis à une politique d'assimilation. Cet article analyse les romans en langue kvène d'Alf NilsenBørsskog comme des contre-récits qui accordent un rôle important à la description de la nature et de l'environnement de la vie quotidienne des Kvènes. On trouve des représentations de Kvènes dans la littérature norvégienne depuis les années 1870, mais la série romanesque Elämän jatko (La continuation de la vie, 2004-2015) de NilsenBørsskog est la première ouvre à raconter leurs expériences et leur environnement du point de vue d'un natif. Elle constitue un contre-récit exceptionnel qui place l'origine de l'auteur au point focal au lieu de représenter la crise d'une culture minoritaire. La description de la nature arctique qu'elle donne est sensiblement différente par rapport aux ouvres précédentes où elle est souvent représentée comme une scène d'expériences extrêmes et de catastrophes et les Kvènes et Sames deviennent l'objet d'une description exotisante. En revanche, Nilsen-Børsskog décrit la vie quotidienne et spirituelle des Kvènes où la nature et l'environnement culturel coexistent en harmonie et forment une partie importante de leur identité.

\section{ABSTRACT}

The Kvens are a national minority in Norway long subject to a policy of assimilation. This article examines the Kven-language novels of AlfNilsen-Børsskog as counter-narratives and descriptions of Arctic nature and the Kvens' living environment. Norwegian fiction has depicted the Kvens since the 1870s, but Nilsen-Børsskog's series Elämän jatko 1-4 (Life Goes On, 2004-2015) is the first to assume a native perspective to the Kvens' living environment and experiences. It forms an exceptional counterstory, focusing on the power of the writer's native background instead of describing a crisis in the minority culture. The descriptions of arctic nature in Life Goes On differ significantly from previous ones, where Arctic it is often depicted as a place of extreme experiences and catastrophes. In addition to nature, the Kvens and Sami have been subjected to this exoticising description. NilsenBørsskog instead describes the Kvens' everyday and spiritual life, in which nature and the cultural environment are in harmony and form an important part of Kven identity.

* Maître de conférences, UiT, Norges arktiske universitet Tromsø. 
Lobjectif de cet article est d'analyser la série romanesque Elämän jatko ( $\mathrm{La}$ continuation de la vie, 2004-2015) de l'auteur kvène Alf Nilsen-Børsskog (19282014) comme un contre-récit ${ }^{1}$ qui accorde un rôle important à la description de la nature et de l'environnement de la vie quotidienne. La série consiste en quatre volumes : Kuosvaaran takana (Derrière la colline Kuosvaara, 2004), Aittiruto $^{2}$ (2007), Rauha (La paix, 2011) et Viimi vuodet (Les dernières années, 2015). Le récit commence à l'automne 1944, vers la fin de l'occupation allemande de la Norvège, au moment où l'armée allemande décide de déporter toute la population du nord de la Norvège hors de la zone de guerre. Pour échapper à cette mesure, les habitants d'un petit village kvène s'enfuient dans les montagnes. Les deux premiers volumes racontent cette fuite et d'autres expériences que les civils ont vécues pendant la guerre. Le troisième volume, Rauha, décrit la période de la reconstruction et la vie des personnages principaux jusqu'aux années 1960. Les décennies 1970-2010 sont couvertes par le dernier volume, Viimi vuodet.

Nilsen-Børsskog est le premier auteur à avoir écrit des romans en kvène, langue finno-ougrienne menacée de disparition, et le premier à décrire les Kvènes du point de vue d'un natif. L'importance du lieu, de la maison et de la nature dans la construction identitaire ainsi que la recherche de l'harmonie dans un sentiment d'appartenance engendré par la culture constituent les thèmes centraux de son œuvre. Elämän jatko est un contre-récit qui ne décrit pas la crise d'une culture minoritaire ou un conflit avec un groupe majoritaire, mais la force de la culture propre des Kvènes.

La notion de " contre-récit " provient des études culturelles postcoloniales où elle a servi à désigner les représentations créées par des groupes - ethniques ou autres - qui se trouvent dans une position de subalterne minoritaire et marginalisé. Les études littéraires utilisent également la notion de " description native " qui souligne l'importance du savoir concernant l'objet de la représentation que l'auteur reçoit de son origine. Selon Gayatri Spivak, un des théoriciens les plus influents dans les études culturelles postcoloniales, seul un membre d'un groupe marginalisé peut créer un vrai contre-récit. Elle soutient que les autres, ceux qui sont extérieurs à ce groupe, n'ont pas les moyens de comprendre la vie, les pensées et les objectifs des marginaux Même s'ils pensent être neutres, ils ne peuvent pas se distancier de leur condition et transposent dans leurs descriptions leurs

1 De l'anglais «counter-narrative» ou «counter-image».

2 Nom propre; les noms propres qui servent de titre ne sont pas traduits. Note du traducteur. 
propres hiérarchies culturelles ${ }^{3}$. Pour Spivak, les récits produits par les membres de groupes marginalisés sont le seul moyen pour faire entendre leur voix ${ }^{4}$.

Bien évidemment, une œuvre écrite par un membre d'un groupe marginalisé n'est pas automatiquement un contre-récit. L'origine de l'auteur n'est que la condition initiale. Pour simplifier, on peut dire qu'une œuvre devient un contrerécit lorsqu'elle rend visibles les valeurs d'un groupe minoritaire et pluralise l'image publique dont il a été l'objet 5 .

Le récit kvène de Nilsen-Børsskog s'ancre solidement dans l'histoire et la société. Avant d'analyser la série Elämän jatko, je vais la situer dans le contexte de l'évolution du statut minoritaire des Kvènes et de leur représentation dans la littérature norvégienne. Les exemples proviennent des œuvres publiées par Jonas Lie, Konrad Dahl, Laura Kieler, J. A. Friis, Henrik Ibsen, Knut Hamsun et Idar Kristiansen.

\section{DE LA MARGE VERS LE STATUT DE MINORITÉ NATIONALE}

Les Kvènes ont acquis le statut de minorité nationale en 1999 et la langue kvène a été reconnue comme langue minoritaire en 2005. Les Kvènes, qui sont d'origine ethnique finnoise, habitent dans le nord de la Norvège au moins depuis le $\mathrm{XVI}^{\mathrm{e}}$ siècle, période où les frontières entre les États modernes n'étaient pas encore tracées. La catégorisation des Kvènes comme une minorité ethnique est produite par l'idéologie du romantisme national du XIX ${ }^{\mathrm{e}}$ siècle qui encourageait la création d'une identité nationale fondée sur une langue commune et une culture unifiéé.

Vers la fin du XIX ${ }^{e}$ siècle, en Norvège, les brumes du romantisme national donnèrent naissance à une pensée raciale qui, appliquée à la politique concernant la citoyenneté, dégrada la condition des Kvènes, Sames, Juifs et Roms. L'unité culturelle fut recherchée dans un climat qui promouvait l'idée de la supériorité de la race norvégienne ou aryenne. Selon cette conception raciste (qu'on peut aussi entendre au sens de racisme culturel), les minorités ethniques étaient catégorisées comme des groupes problématiques de rang inférieur à intégrer sans tarder à

3 Gayatri Spivak, "Subaltern Studies : Deconstructing Historiography ", in The Spivak Reader. Selected Works of Gayatri Chakravorty Spivak, D. Landry et G. MacLean (dir.), New York, Routledge, 1996, p. 205-209.

4 Gayatri Spivak, "Can the Subaltern Speak ? ", in Marxism and the Interpretation of Culture, C. Nelson et L. Grossberg (dir.), Urbana, University of Illinois Press, 1988, p. 282, 293.

5 Voir par exemple David Huddart, Postcolonial Theory and Autobiography, Londres, Routledge, 2008 ; Gayatri Spivak, "Can the Subaltern Speak ?", op. cit.

6 Kari Storaas, "Fra innvandrer til nasjonal minoritet ", Arina. Nordisk tidsskrift for kvensk forskningPohjoismainen kveenitutkimuksen aikakausjulkaisu 2009-2010, vol. 7, 2010, p. 104-105 ; Knut Einar Eriksen, Einar Niemi, Den finske fare. Sikkerhetsproblemer og minoritetspolitikk i nord 1860-1940, Oslo, Universitetsforlaget, 1981, p. 30-31. 
la majoritét. En accusant les Kvènes d'isolement, d'attachement obstiné à leur langue et à leurs mœurs, et de pratiquer le laestadianisme, on leur assigna l'image d'ennemi culturel ${ }^{8}$. On soupçonnait également les Kvènes de ne pas avoir de sentiment patriotique à l'égard de la Norvège'. D'autre part, ils vivaient paisiblement dans leurs villages et se rendaient utiles à la vie économique grâce à leur savoirfaire dans l'agriculture arctique, l'élevage, la pêche, et la petite industrie. L'espace vital ne manquait pas; le gouvernement voulait en réalité avoir plus d'habitants dans le nord de la Norvège pour renforcer sa présence dans les zones frontalières ${ }^{10}$. On n’a donc pas procédé à des déportations, mais décidé d'assimiler les Kvènes aux Norvégiens.

Ces conceptions irriguent la plus grande partie des œuvres littéraires norvégiennes où on trouve des Kvènes ${ }^{11}$. Même la fiction souligne l'altérité, la primitivité et l'inconfort dans l'image qu'elle donne de ce groupe ethnique. Les Kvènes avaient de faibles chances de participer à l'élaboration de leur propre image. Ils habitaient loin des centres de pouvoir et n'avaient pas de classe cultivée ni d'élite politique ou économique ; leurs institutions n'existaient pas. Pour ces raisons, les écrits publiés par des auteurs et fonctionnaires norvégiens ont constitué pendant longtemps le fondement des conceptions culturelles sur les Kvènes.

L'évolution inquiétante du discours politique sur la nation et la citoyenneté explique le long silence des Kvènes dans la sphère publique. Ce n'est que dans les années 1980 que les Kvènes, encouragés par les mouvements ethnopolitiques globaux et l'évolution de la société, ont commencé à exiger du pouvoir central des mesures pour renforcer leur langue et leur culture. Les revendications les plus importantes, dont certaines ont depuis été satisfaites, concernaient la création d'institutions culturelles et de médias propres, l'enseignement de la langue à l'école, et la participation aux prises de décisions politiques ${ }^{12}$. En 1999, la Norvège a ratifié

7 Torgeir Skorgen, Rasenes oppfinnelse. Rasetenkningens historie, Oslo, Spartacus, 2002, p. 189-191 ; Anne-Lise Seip, "Det norske "vi" : kulturnasjonalisme i Norge ", in Jakten på det norske. Perspektiver på utviklingen av en norsk nasjonal identitet på 1800-tallet, Øystein Sørensen (éd.), Oslo, Ad Notam Gyldendal, 1998, p. 103-104.

8 Rolf Inge Larsen, Religion og fiendebilder. Lastadianismen, statskirken og kvenene 1870-1940, Tromsø, Universitetet i Tromsø, 2012, p. 20.

9 Kari Storaas, "Fra innvandrer til nasjonal minoritet », op. cit., p. 124.

10 Knut Einar Eriksen, Einar Niemi, Den finske fare, op. cit., p. 127-130.

11 Voir par exemple Anitta Viinikka-Kallinen, "Kaunokirjallisuus kansallisuuspolitiikan palveluksessa. Etnisyyden representaatiot J. A. Friisin tuotannossa ", Kirjallisuudentutkimuksen aikakauslehti Avain. The Finnish Review of Literature Studies, 3/2015, p. 34-50 ; et Anitta Viinikka-Kallinen, "Kulttuuri kriisinhallinnan keinona : Alf Nilsen-Børsskogin romaani luo kveenien vastahistoriaa ", in Ódagumeresoomõ kodo. Läänemeresoome kodu, H. Koks et J. Rahman (éd.), Vốro, Vốro Instituut (Vớro instituudi toimóndusóq ; 20), 2007, p. 107-119.

12 Ce travail est porté en particulier par l'association Norkse Kveners Forbund fondée en 1987, voir http://kvener.no/kv/kvensk-hovedside/. 
le traité du Conseil de l'Europe qui reconnaît le statut de minorité nationale des Kvènes et oblige l'État à protéger et promouvoir leur culture.

Être l'objet d'une longue et dure politique d'assimilation fut une expérience traumatisante pour les Kvènes. La discussion publique ne l'a abordé que petit à petit ; le travail de deuil ne vient que de commencer ${ }^{13}$. Sur un plan psychologique, on estime généralement que les individus et les communautés confrontés à des situations traumatisantes ont besoin de beaucoup de temps avant qu'ils ne puissent traiter l'expérience et la surmonter, la première obligation après le choc étant de survivre et retrouver la capacité à agir. Ce n'est que dans un deuxième temps que la communauté traumatisée veut se souvenir et comprendre pour pouvoir construire son avenir ${ }^{14}$. Dans les années 2000, les Kvènes ont commencé à exiger qu'on remémore les événements historiques et qu'ils soient documentés, et que le gouvernement reconnaisse les injustices commises dans le passé et les répare en leur octroyant à présent des droits.

Une des missions de la littérature est d'aider une communauté à se souvenir de son passé et à l'analyser. La série romanesque de Nilsen-Børsskog l'accomplit en racontant l'histoire des Kvènes des années 1940 jusqu'à aujourd'hui. Par son art, un auteur peut exprimer des sujets que la recherche historique et les enquêtes ne révèlent pas. La représentation publique des expériences et sentiments individuels constitue une part importante dans le rétablissement d'une communauté traumatisée.

\section{LES KVÈNES ENTRE MYTHES SUR L'ARCTIQUE ET CLICHÉS DE FICTION}

Les descriptions des régions septentrionales offrent de nombreux exemples sur la représentation de personnes et conditions de vie par des auteurs extérieurs qui semblent tout ignorer à leur sujet. L'extrême nord de l'Europe a été l'objet de nombreux récits de voyage et rapports rédigés par des fonctionnaires depuis le Moyen Âge, le XIX ${ }^{\mathrm{e}}$ siècle étant une période particulièrement riche en textes publiés dans plusieurs langues. La maitrise que les auteurs ont de leur sujet varie beaucoup, mais les écrits partagent la même tendance à souligner l'altérité, c'està-dire à représenter "l'Arctique " comme un écart à la norme et structurellement différent par rapport au prétendu monde civilisé. L'attention des auteurs a été attirée en particulier par la longue distance qui sépare la région des centres du pouvoir ainsi que par la nature dramatisée grâce à une description qui a cherché à mettre en valeur ses caractéristiques extrêmes et à la présenter comme le théâtre de

13 Anitta Viinikka-Kallinen, «While the wings grow : Finnic minorities writing their existence onto the world map ", in Planning a new standard language. Finnic minority languages meet the new millennium, H. Sulkala et H. Mantila (éd.), Helsinki, SKS, 2010, p. 147-177, p. 164.

14 Antonius C. G. M. Robben, «How Traumatized Societies Remember », Cultural Critique, no 59, 2005, p. 120-164, p. 121-123. 
catastrophes et luttes exaltantes. Une imagerie appropriée à ces besoins était fournie par l'hiver long et noir avec sa nuit polaire, l'été court avec sa lumière, l'océan Arctique avec ses baleines et phoques et le désert enneigé avec ses montagnes ${ }^{15}$.

Ces particularités du monde naturel semblent avoir incité les auteurs à interpréter la culture et les êtres humains d'une manière exotisante. Parfois, cette perception a donné lieu à des approches romantisantes, mais plus souvent à des descriptions discriminatoires relevant même du racisme culturel. Les Kvènes et les Sames ont fini par représenter l'exotique dans les textes consacrés aux régions septentrionales de la Norvège ${ }^{16}$. Le mode de vie, les moyens de subsistance et l'apparence physique des Sames en particulier ont été décrits dans un esprit de racisme culturel débridé dans des récits de voyage. Enrichir leurs connaissances ne semble pas avoir été l'objectif des auteurs qui ont plutôt cherché des sujets qui leur ont paru étrangers et dont la description était fondée sur des conceptions préexistantes. La dimension intertextuelle est si importante qu'on peut parler de mythes qui ont transité d'une œuvre à l'autre ${ }^{17}$.

Vers la fin du XIX ${ }^{\mathrm{e}}$ siècle, la littérature norvégienne voit la publication de nombreux ouvrages dont le récit se situe dans le nord du pays. Le choix de ce sujet était probablement influencé par les nombreux récits de voyage qui ont vu le jour suite au progrès technologique qui rendait les déplacements plus faciles ${ }^{18}$. Dans ces ouvrages, les personnages principaux viennent souvent d'ailleurs pour un séjour dans le Nord dont la durée peut varier entre quelques semaines et plusieurs décennies; ils font quelques observations superficielles et constatent les particularités des conditions de vie et des habitants de la région ; à la fin, ils quittent l'Arctique qui reste à leurs yeux un territoire d'exception. La description du milieu ressemble aux récits de voyage. Le narrateur typique se résume à un œil extérieur qui observe les conditions de vie exotiques et les hommes étranges qui y habitent.

Les Kvènes commencent à apparaitre dans la littérature norvégienne à partir des années $1870^{19}$, bien que la plupart des œuvres publiées durant cette période ne

15 Kari Aga Myklebost, Borealisme og kulturnasjonalisme. Bilder av nord $i$ norsk og russisk folkeminnegransking 1830-1920, Tromsø, Universitetet i Tromsø, 2010. p. 8.

16 Silke Göttsch-Elten, "Populäre Bilder vom Norden im 19. und 20. Jahrhundert ", in Ultima Thule. Bilder des Nordens von der Antike bis zur Gegenwart, A. Engel-Braunschmidt, G. Fouquet, W. von Hinden et I. Schmidt (éd.), Francfort-sur-le-Main, P. Lang, 2001, p. 124 ; Anitta Viinikka-Kallinen, "Kaunokirjallisuus kansallisuuspolitiikan palveluksessa », op. cit.

17 Veli-Pekka Lehtola, Rajamaan identiteetti. Lappilaisuuden rakentuminen 1920-ja 1930-luvulla kirjallisuudessa, Helsinki, SKS, 1995, p. 47-49.

18 Le réseau de transports maritimes réguliers a été étendu vers le nord pour couvrir les villes côtières les plus septentrionales vers la fin du XIX ${ }^{\mathrm{e}}$ siècle, voir Erling Storrusten, Hurtigruten. Verdens vakreste sjøreise, Narvik, Hurtigruten ved Ofotens og Vesteraalens dampskibsselskab AS, 1999, p. 6-7.

19 May Molund, Sverre Pedersen, Kvenene i litteraturen, Hovedoppgave i nordisk litteratur, Tromsø, Universitetet i Tromsø, 1982, p. 97 ; Anitta Viinikka-Kallinen, « While the wings grow », op. cit., p. 164. 
puissent pas être qualifiées de description des Kvènes. Les auteurs reconnaissent les Kvènes et savent qu'il s'agit d'un groupe ethnique à part, mais ils leur offrent le rôle de personnages secondaires caricaturaux et ne semblent pas en savoir plus à leur sujet. Les récits commencent très tôt à recycler quelques clichés intertextuels dont l'origine remonte à la discussion publique et à la littérature antérieure. L'image est pauvre et unidimensionnelle ; le Kvène est primitif par son apparence physique et son caractère, il est un laboureur monotone au comportement renfrogné et menaçant. Doté de pouvoirs surnaturels et d'une sexualité débordante, il pratique la religion d'une manière fanatique.

L'image est morose, mais il faut se rappeler, quand on étudie la littérature de cette période, que les stratégies narratives ethnifiantes étaient largement acceptées et utilisées au XIX ${ }^{e}$ siècle. La présentation et la classification des caractéristiques ethniques étaient déjà présentes dans la littérature du Moyen Âge. Quand les langues nationales ont commencé à supplanter le latin, les différences culturelles et ethniques sont devenues plus visibles et le besoin de catégoriser les êtres humains plus pressant ${ }^{20}$. Ces descriptions qui ne sauraient être publiées aujourd'hui étaient monnaie courante. Les représentations ethnifiantes ont été l'objet de critique dans la recherche postcoloniale, en particulier en raison de leurs contenus racistes et discriminatoires ${ }^{21}$. En même temps, la recherche a souligné l'importance des contre-récits et de la voix propre du groupe marginalisé22.

Dans la littérature du XIX ${ }^{e}$ siècle, la fonction des Kvènes est ainsi d'apporter dans le texte une couleur locale et des tensions ethniques. On peut cependant trouver quelques exceptions intéressantes à ce modèle, comme le roman Eda Mansika (1875) de Konrad Dahl, dont le personnage principal éponyme est une jeune femme kvène. Elle est intelligente, énergique, a de l'assurance et prend des résolutions courageuses. Le personnage principal est kvène dans le roman de Laura Kieler Lavrekas Korhoinen (1881) publié au Danemark, mais le récit est un mélange confus de description de nature romantique, de récits sinueux et de mysticisme. Kieler confond les Kvènes et les Finnois, par mégarde ou délibérément.

Une femme kvène nommée Anne Kven apparaît dans le roman Den fremsynte, eller Billeder fra Nordland (Le clairvoyant, ou images du Nordland, 1870) de Jonas Lie, un des auteurs classiques de la littérature norvégienne. Anne est une servante renfrognée et hommasse qui fume la pipe d'un air morose, mais dont

20 Joep Leerssen, « The poetics and anthropology of national character (1500-2000)», in Imagology. The cultural construction and literary representation of national characters. A critical survey, M. Beller et J. Leerssen (éd.), New York - Amsterdam, Rodopi, 2007, p. 64-66.

21 Abdul JanMohamed, "The Economy of Manichean Allegory : The Function of Racial Difference in Colonialist Literature », in "Race», Writing, and Difference, H. L. Gates Jr. (éd.), Chicago, University of Chicago Press, 1986, p. 78-106. p. 83-88.

22 Gayatri Spivak, "Can the Subaltern Speak ? ", op. cit., p. 293 ; David Huddart, Postcolonial Theory and Autobiography, op. cit., p. 125-126. 
l'attachement à sa maîtresse est émouvant. La magie et les forces invisibles sont des motifs importants dans l'œuvre qui crée une image mythique du Nord et de ses habitants. Anne Kven représente les puissances métaphysiques qui sont centrales pour la thématique du roman ; son altérité ethnique souligne sa particularité.

Le roman à succès de J. A. Friis Fra Finmarken. Skildringer (Du Finnmark. Descriptions, 1881), connu plus tard sous le titre Lajla, est un récit d'aventures palpitant et romantique où la nature aride de l'Arctique constitue le fond de la vie dramatique d'une jeune fille same. Professeur de langues same et kvène, Friis porta souvent les problèmes des Sames à la connaissance publique. Ses activités montrent cependant une conception fondée sur la hiérarchie des races où les Sames sont inférieurs aux Norvégiens. Les représentations des Sames dans sa production littéraire sont à la fois romantisantes et discriminatoires. Il décrit les Kvènes comme des représentants d'un mal quasi métaphysique. Dans Lajla, ils sont querelleurs, violents, malhonnêtes, et même laids ${ }^{23}$. Le livre a eu une grande influence sur la perception des groupes ethniques en Norvège. Il a été édité quinze fois et traduit dans de nombreuses langues ${ }^{24}$. Le succès repose probablement sur le fait que le roman de Friis reproduit des mythes et clichés sur les régions arctiques et les Sames sous forme d'un récit divertissant et facile à lire.

Henrik Ibsen et Knut Hamsun, deux grands auteurs de la littérature nationale norvégienne, ont aussi intégré des Kvènes dans leurs ouvres. Dans la pièce de théâtre Fruen fra havet (La dame de la mer, 1888) d'Ibsen, le Kvène est un inconnu mystérieux qui débarque d'un navire étranger et dont le charme magique est irrésistible au personnage principal, Ellida. Le Kvène persuade Ellida de quitter son mari et partir à l'étranger. Plus tard, on apprend qu'il porte un faux nom parce qu'il a tué le capitaine d'un autre navire et qu'il fuit la police. L'altérité ethnique souligne son extériorité et sa répugnance à respecter la loi et les normes morales de la société.

Dans le roman Mysterier (Mystères, 1892) de Hamsun, le personnage principal, Johan Nilsen Nagel, est un intellectuel névrotique qui arrive dans une petite ville côtière et crée la confusion avec son mode de vie particulier. Nagel affirme être Kvène et originaire du Finnmark. Le motif de l'ethnicité apparaît hâtivement, mais l'information est importante pour la thématique du roman. L'inquiétude et l'absence de racines sont des traits fondamentaux du personnage de Nagel qui s'expliquent partiellement par son altérité ethnique. Comme le Kvène d'Ibsen, il a une morale douteuse et ne se conforme pas aux normes de la société. De plus, il est autodestructeur et se suicide à fin du roman.

23 Anitta Viinikka-Kallinen, "Kaunokirjallisuus kansallisuuspolitiikan palveluksessa », op. cit., p. 37.

24 En français Lahila. Récits et études de moeurs de la Laponie norvégienne (Lausanne, Payot, 1895) et Lajla. Roman du pays lapon (Paris, Je sers, 1943). 
À partir des années 1920, la présence des Kvènes a diminué et même presque cessé, avant que le sujet ne revienne au premier plan avec la série romanesque Kornet og fiskene 1-4 (Le grain et les poissons, 1978-1981) d'Idar Kristiansen. Elle décrit la situation désespérée des habitants de la région de Kainuu en Finlande pendant la grande famine des années 1860 et la nouvelle vie du personnage principal en Norvège. Les Kvènes ne sont pas le véritable sujet de Kristiansen, mais plutôt les immigrés finlandais de la première génération. Les romans ont suscité un vif intérêt et une discussion sur la vraisemblance de l'image de l'histoire qu'ils offrent $^{25}$, ce qui montre qu'un sujet lié aux Kvènes intéressait toujours le public.

On peut ainsi trouver des Kvènes ici et là dans la littérature norvégienne, mais leur propre voix en a été longtemps absente. La connaissance qu'en ont les auteurs non kvènes est peu profonde ; ils ignorent la culture kvène et ne parlent ni le kvène ni le finnois. Friis est l'exception, mais il ne met pas à profit son érudition dans ses œuvres littéraires.

\section{TOUT EST DIFFÉRENT DANS UN CONTRE-RÉCIT}

Le rôle des Kvènes dans la littérature semble ainsi peu évoluer jusqu'au grand tournant que représente la série Elämän jatko d'Alf Nilsen-Børsskog. Il abandonne les catégorisations utilisées auparavant et aborde des sujets importants pour les Kvènes, dont la maison et la famille, l'amour de la nature et de la culture ainsi que les valeurs éthiques de la communauté.

Au début de la série, un petit groupe de Kvènes fuit les déportations dans les montagnes pour vivre dans une kammi, une hutte couverte de tourbe. Le groupe est composé d'hommes et de femmes d'âges différents et de quelques enfants ; les deux premiers volumes racontent la fuite sans accorder de rôle principal à aucun des personnages.

Les fugitifs se trouvent dans une situation menaçante. Il n'y a pas beaucoup de nourriture et le froid s'installe; l'absence d'information sur le sort des proches et l'évolution de la guerre pèsent sur les esprits. La survie dans les conditions arctiques devient le principal défi. Dans le chaos de la guerre, la sécurité offerte par la culture et les normes morales de la communauté est le salut de l'âme des individus et du groupe, et le savoir-faire lié à la nature leur permet de survivre. Dans Elämän jatko, la nature arctique n'est pas un terrain exotique pour des aventures, mais un refuge qui permet de se mettre à l'abri d'une menace extérieure. Le rapport des fugitifs à la nature évolue dans des conditions critiques. Les croyances

25 Kaisa Maliniemi Lindbach, Kvenlitteratur i nord. Med spesielt blikk på Idar Kristiansens romaner, thèse de doctorat, Tromsø, Universitetet i Tromsø, 2001, p. 5-29. 
folkloriques en des esprits maléfiques qui guettent leur proie dans le désert disparaissent ; seule l'action humaine apparaît désormais comme dangereuse :

Mettän pahat henget, joita het ennen rauhanaikana olthiin niin pölanheet [...] ei ennää näyttänheet heile niin vaaralisilta ja pölättäviltä. Pimmee, pahat henget ja vielä mettän elläimetki, tulthiin luotettaviksi ja ystävälisiksi olenoiksi, joita kukhaan ei tarvinu pölätä. Ihmistä, sen sijhaan, piti pölätä ja siitä piti karata. ${ }^{26}$

Les mauvais esprits de la forêt, dont ils avaient eu peur avant la guerre [...] ne leur semblaient plus si dangereux et menaçants. Le noir, les mauvais esprits, et même les animaux sauvages étaient devenus des êtres fiables et amicaux qu'on ne devait pas craindre. Par contre, on devait avoir peur de l'homme et le fuir.

Iisakki, un jeune père de famille, est un des fugitifs. Il se sent coupable de la déportation de sa famille. Philosophe du groupe, il parle souvent de la morale, de la justice et du déclin de la civilisation. Pour lui, la guerre est la preuve de l'impuissance de la culture face au mal. Être dans la nature soulage les tourments de l'esprit et rétablit la foi en la vie.

Raskhaat ajatukset ja syylisyyden tunnet keveni korkkeela, puunrajan ylipuolela. Tunturin lajela mies eläskeli parhaimat hetket. [...] Monta kertaa oli ihana ja suloinen autuuden tunnet täyttäny hänen sielun [...] ilola jota hän muvvala ei muistanu tuntenheen. Kuvvaamaton rakhaus oli näinä hetkinä tulvinu onnettoman miehen sielhuun ja sydämheen. ${ }^{27}$

Les pensées accablantes et la culpabilité devenaient plus légères en hauteur, au-dessus de la limite des arbres. Au sommet de la colline, l'homme a vécu les meilleurs moments de son existence. [...] De nombreuses fois, un sentiment de béatitude doux et délicieux avait envahi son âme [...] avec une joie qu'il ne se rappelait pas avoir sentie ailleurs. Un amour indescriptible avait alors rempli l'âme et le cœur du pauvre homme.

La vie paisible du village kvène a été brutalement interrompue par la guerre. La civilisation lutte contre la barbarie. Le narrateur met en question la conception hiérarchique conventionnelle en constatant que le mal qui menace la vie des Kvènes et de tous les habitants de la Norvège du Nord vient de l'ancien berceau de la civilisation, l'Allemagne. Aux yeux des fugitifs, elle représente désormais la violence et la déchéance morale. Tout le monde est au courant des mauvais traitements, de la violence, et même des meurtres dont les prisonniers et les civils sont l'objet. Les fugitifs en parlent aussi :

Ei muuta ko kurzen Prozess mit dem Gefangenen machen. Lyhyt prosessi vain ja miestä ei ennää ollu olemassakhaan. Luoti vain niskakuophaan. Ei ollu moraaliikhaan. Suuresti sivistynheen tyskän kansan moraali oli pudonu turpheen tasala. Niin ko paljot Finnmarkun talotki ${ }^{28}$

26 Alf Nilsen Børsskog, Elämän jatko 1. Kuosuvaaran takana, Indre Billefjord, Idut, 2004, p. 30-31.

27 Ibid., p. 360.

28 Ibid., p. 23. 
Rien d'autre que kurzen Prozess mit dem Gefangenen machen ${ }^{29}$. Juste une courte procédure et l'homme n'exista plus. Juste une balle dans la nuque. Pas de morale. La morale du peuple allemand hautement civilisé était tombée au niveau de la tourbe. Comme de nombreuses maisons dans le Finnmark!

Nilsen-Børsskog représente la nature arctique comme le foyer de l'homme, son refuge et la source de sa subsistance. Elle offre de la nourriture et des expériences esthétiques, soulage dans les épreuves et sauve littéralement la vie des Kvènes. Selon le géographe Yi-Fu Tuan, la division binaire entre la culture et la nature qu'on trouve souvent dans l'art et dans la recherche a tendance à produire une catégorisation rigide des lieux en centres civilisés et périphéries primitives ${ }^{30}$. Le contre-récit de Nilsen-Børsskog rompt cette conception de nature binaire et traditionnelle. Dans son œuvre, la nature n'est pas un espace périphérique pour la récréation de l'homme qui quitterait occasionnellement son milieu culturel. L'auteur met en scène une relation presque symbiotique entre la nature et l'homme qui est la condition pour maintenir la capacité à agir, la paix de l'âme et un mode de vie éthique et juste.

Le terme " contre-récit " peut laisser penser que la série romanesque contiendrait de nombreux thèmes orientés contre les anciennes descriptions discriminatoires, et même qu'un récit raconté par la voix propre d'un auteur kvène se concentrerait à prendre le contrepied des représentations négatives. Dans ce cas, le contre-récit serait conditionné par des contenus qu'il cherche à mettre en question et renverser. On peut justifier une telle interprétation si l'on conçoit la littérature avant tout comme un continuum où les nouvelles œuvres se construisent sur les anciennes et entretiennent un dialogue intertextuel avec elles. Comme je l'ai démontré plus tôt, les lignes directrices traditionnelles de la représentation des Kvènes dans la littérature norvégienne semblent en effet résulter d'un tel continuum ; la même observation vaut pour la représentation des Sames ${ }^{31}$. Un auteur qui crée un contre-récit peut cependant choisir n'importe quelle approche et mode de narration. N'ayant pas d'obligation de se positionner par rapport aux textes précédents, il peut traiter aussi des thèmes qui sont absents de la tradition. La littérature entretient une relation dialogique non seulement avec sa propre tradition, mais également avec d'autres discours; elle communique avec la réalité et en crée de nouvelles ${ }^{32}$.

Une interprétation exclusivement focalisée sur la critique des structures hiérarchiques et discriminatoires peut mener à une cécité par rapport à la richesse de

29 "Suivre la procédure courte avec les prisonniers », en allemand dans le texte. Note du traducteur.

30 Yi-Fu Tuan, "Cultural Geography. Glances Backward and Forward », Annals of the Association of American Geographers, vol. 94 (4), 2004, p. 731-732.

31 Veli-Pekka Lehtola, Rajamaan identiteetti, op. cit., p. 47-49.

32 Pertti Karkama, Kirjallisuus ja nykyaika. Suomalaisen sanataiteen teemoja ja tendenssejä, Helsinki, SKS, 1994, p. 8-9. 
l'œuvre analysée ${ }^{33}$. Nilsen-Børsskog ne crée par une configuration binaire « nous vs. eux ». Ses romans se situent directement dans le contexte de leur propre grand récit. L'identité kvène n'est pas créée par la négation, mais par une conscience de soi fondée sur la fierté de sa propre culture. Le mode narratif qui met en valeur la force et l'habileté des Kvènes semble se justifier par un choix conscient d'éviter la thématique de la victimisation, c'est-à-dire d'éviter de donner une image des Kvènes comme victimes des circonstances. Dans sa représentation des Kvènes, Nilsen-Børsskog dirige l'attention vers d'autres sujets que ceux abordés par les auteurs norvégiens régulièrement focalisés sur leur altérité problématique qui serait liée à la langue et l'ethnie.

Dans les anciennes descriptions ethnifiantes, les personnages kvènes restent superficiels et schématiques. Une des raisons en est que la littérature et la discussion publique offraient de nombreux modèles intertextuels pour de telles descriptions. La représentation d'un groupe ethnique qui habite dans le même pays que l'auteur, mais qui lui est inconnu, est un défi. La recherche a désigné cette problématique par les notions de "l'autre intérieur" (the other within) et "l'autre domestique "(domestic other) ${ }^{34}$. Un exemple de cette problématique est la représentation des Roms dans la littérature européenne. Ils constituent un groupe large et visible dont on trouve de très nombreuses descriptions ${ }^{35}$, tandis que les Kvènes forment un petit groupe territorial situé dans le nord de la Norvège. Le mécanisme derrière les descriptions ethnifiantes semble cependant être le même. On sélectionne quelques caractéristiques réelles ou imaginaires de l'objet de la représentation pour en construire un personnage fictif. Dans la littérature britannique par exemple, la représentation des Roms s'est appauvrie déjà au XVIII ${ }^{e}$ siècle pour ne répéter que quelques stéréotypes comme l'altérité raciale, le code vestimentaire particulier, le mode de vie nomade, le primitivisme, la criminalité et la sexualité débridée ${ }^{36}$.

Dans sa série romanesque, Nilsen-Børsskog élève les Kvènes au point focal de la narration et ne les compare pas avec les autres. Il n'endosse pas le rôle d'écrivain minoritaire, mais travaille comme un auteur national ; il décrit son propre groupe ethnique de l'intérieur et sans comparaison. Les autres groupes ethniques du nord de la Norvège - les Sames et les Norvégiens - ne sont pas non plus l'objet d'une

33 Leela Gandhi, Postcolonial Theory. A Critical Introduction, $2^{e}$ éd., New York, Columbia University Press, 2019, p. 167-168.

34 Viola Parente-Čapková, " Kotimainen toinen. Romanit suomalaisuuden kirjallisena rakennusaineena ", Kulttuurintutkimus, vol. 28, no 2, 2011, p. 3-18, p. 3-4.

35 Colin Clark, "Severity has often enraged but never subdued a gypsy. The History and Making og European Romani Stereotypies ", in The Role of the Romanies. Images and counter-images of "Gypsies"I Romanies in European cultures, N. Saul et S. Tebbutt (éd.), Liverpool, Liverpool University Press, 2004, p. 228-230.

36 Ibid., p. 234-236. 
représentation négative ou marginalisante ; ils apparaissent comme appartenant à la région multiculturelle de Pohjoiskalotti ${ }^{37}$. Nilsen-Børsskog ne fait pas d'ethnification, mais donne des traits de caractère individuels à ses personnages. Libérés du sentiment d'altérité, les Kvènes ne se sentent pas étrangers dans leur propre pays ; ils se considèrent membres du peuple norvégien : "Miksi justhiin met pidämä elläät tämmäistä vainon ja rauhattomuuden aikkaa? Mitä pahhaa Norjan kansa on tehny, ette tämmäinen rangaistus on langennu meidän niskoile? huokkaili emäntä » (" Pourquoi devons-nous vivre un tel temps de persécution et d'inquiétude ? Quel mal a commis le peuple norvégien pour qu'un tel châtiment tombe sur nous? " soupira la fermière).

Le conflit entre le bien et le mal est central dans Elämän jatko. Aucun être humain ou groupe ethnique n'est cependant représenté comme structurellement mauvais. Le mal apparaît comme résultant d'un écart par rapport à la situation normale et ses causes sont soumises à une analyse philosophique. L'armée nazie incarne la violence et le chaos. Le moyen de défense des fugitifs face à la haine et la violence est de préserver la dimension éthique de leur vision du monde.

\section{LA RÉPONSE DANS LA NATURE}

Nilsen-Børsskog considère qu'une relation étroite avec la nature est la source de l'équilibre mental de l'homme, sans pour autant accorder de signification métaphysique ou religieuse à la nature. Avoir une relation vivante avec la nature et l'environnement est une nécessité spirituelle et même physique. Dans le troisième volume de la série, la transformation de la société dans les années qui ont suivi la guerre contraint le personnage principal Arne à quitter sa région d'origine. Il travaille et étudie avec succès dans différents endroits en Norvège, mais il est poursuivi par un sentiment d'angoisse qui prend peu à peu la dimension d'un trauma de guerre. Les randonnées dans les montagnes familières et les paysages déserts lui sont indispensables pour qu'il puisse s'apaiser et retrouver son énergie. À la fin, il décide de retourner chez lui et d'en finir avec son vagabondage à travers le monde.

La thématique du départ et du retour est essentielle dans Elämän jatko, où elle est traitée avec les moyens de la littérature moderne. Pour le sociologue Zygmunt Bauman, le malaise existentiel de l'homme contemporain vient du fait qu'il a dû renoncer à l'espoir d'acquérir une identité stable et d'avoir le sentiment d'appartenir à un lieu. La perte d'un foyer authentique est l'expérience fondamentale de l'homme; la confusion et l'anxiété en sont les conséquences ${ }^{39}$. La relation profonde entre le lieu et l'identité constitue bien un motif central

37 "Nordkalloten » en norvégien, la région arctique de la Norvège, la Suède, la Finlande et du nordouest de la Russie. Note du traducteur.

38 Alf Nilsen-Børsskog, Elämän jatko 2. Aittiruto, Indre Billefjord, İut, 2007, p. 258.

39 Zygmunt Bauman, Savnet fellesskap, Oslo, Cappelen Akademisk Forlag, 2000, p. 88-89. 
dans la littérature moderne, dans un contexte plus large aussi. Le dépassement des frontières physiques et spirituelles est une contrainte, d'une part et une possibilité, d'autre part ${ }^{40}$. Nilsen-Børsskog décrit dans ses romans aussi bien l'expérience d'une identité stable et de l'appartenance à un lieu que leur perte traumatique et la recherche de nouvelles possibilités. On peut interpréter la vie et les expériences du personnage principal comme résultant de sa séparation avec son milieu authentique. Au début, les raisons de cette séparation sont la guerre et la transformation de la société, mais au fur et à mesure que la narration avance, le départ apparaît comme la conséquence d'une inquiétude intériorisée.

Le motif du retour à la maison prend une importance particulière dans la littérature des minorités ethniques. Il est souvent relié à la décision de retourner parmi ses semblables et de choisir une identité fondée sur l'appartenance. La nature et un milieu ressenti comme sien renforcent l'homme. Claudia Deetjen a analysé le motif du retour dans la littérature des peuples indigènes des ÉtatsUnis, où il est souvent lié aux expériences traumatiques que les communautés ont vécues, comme la dissolution des liens traditionnels, la paupérisation et d'autres problèmes sociaux. En même temps que les peuples indigènes ont formé des mouvements pour leur émancipation, la littérature a vu l'émergence de récits sur l'expérience d'hommes indigènes traumatisés pour plusieurs raisons, comme les vétérans de guerre, les victimes de déplacement forcé ou les anciens élèves d'internats. Dans ces récits, le retour au milieu originel, plein de significations positives, de symboles d'appartenance et d'harmonie avec la nature, est présenté comme un moyen pour regagner un sentiment d'apaisement ${ }^{41}$.

Les récits de retour de ce type ont été qualifiés par le terme "contre-pastoral " dans la recherche postcoloniale. On considère alors que l'auteur indigène présente un récit qui va à l'encontre de l'imagerie romantique liée aux colons. On peut en trouver des exemples dans la littérature des États-Unis, d'Australie, du Canada et d'Afrique du Sud ${ }^{42}$. Dans ces contre-récits, les descriptions de la nature et de l'environnement culturel rendent visibles des lieux, mémoires et symboles qui ont une importance pour le peuple indigène marginalisé. Dans les célèbres romans La maison de l'aube (House Made of Dawn, 1968) de N. Scott Momaday et La cérémonie (Ceremony, 1977) de Leslie Marmon Silko, le personnage principal retourne, à l'instar d'Arne dans Elämän jatko, du monde urbain vers un milieu qui est en harmonie avec la nature ${ }^{43}$.

40 Ulrich Beck, What is Globalization ?, Cambridge, Polity Press, 2000, p. 75-76.

41 Claudia Deetjen, Re-imagining nature's nation. Native American and native Hawaiian literature, environment, and empire, Heidelberg, Winter, 2016, p. 49-50.

42 Lawrence Buell, «American Pastoral Ideology Reppraised », American Literary History, vol. 1, no 1, 1989, p. 62-66 ; Graham Huggan, Hellen Tiffin, "Green Postcolonialism », Interventions, 9 (1), 2007, p. $82-120$, p. $85-90$.

43 Claudia Deetjen, Re-imagining nature's nation, op. cit., p. 49-50. 
Chez Nilsen-Børsskog, même la rudesse arctique de la nature souligne la fonction d'harmonisation. L'auteur renverse une configuration bien connue : la nature arctique offre ici consolation et équilibre, alors que dans la littérature précédente et dans les récits de voyage elle est menaçante et exotique et sa beauté repose sur une altérité mythique ${ }^{44}$. Dans Elämän jatko, la côte de la mer Arctique et les montagnes du Finnmark sont un foyer qui protège et une source d'harmonie mentale. Les magnifiques dimensions des montagnes, l'eau qui coule en abondance dans les rivières et les immenses déserts rappellent à l'homme sa taille modeste et la relativité de toute chose. Ce n'est que dans son paysage propre, à l'abri des montagnes familières que l'angoisse d'Arne lâche prise.

Il est possible de voir dans Elämän jatko une allégorie sur le destin de la communauté kvène : un voyage d'un état d'harmonie prémoderne vers l'anxiété moderne et la reconstruction d'une nouvelle identité. Une des tâches les plus importantes de la littérature moderne est l'analyse des thèmes existentiels ${ }^{45}$. Jugé avec ce critère, Elämän jatko est bien une œuvre moderniste. En revanche, dans la description de la communauté des Kvènes et leur loyauté réciproque, l'auteur s'approche de l'idylle prémoderne. Dans une société prémoderne, les relations sociales reposent sur le travail et les liens familiaux et la vie est régulée par les cycles de la nature. Nilsen-Børsskog situe la fin de l'idylle au milieu de la catastrophe de la guerre où les gens doivent quitter - littéralement et métaphoriquement leur foyer. La scène initiale de la série décrit son effondrement et la fuite vers un avenir inconnu. Un beau jour d'automne, un groupe de réfugiés marche dans le désert. Ils ont quitté leur village, mais la beauté et la paix immuable de la nature soulagent leurs angoisses : "pikkulinttuin aamulaulu paransi heidän mielen, niin ette unettui missä het olthiin, ja miksi het olthiin tulheet tänne " ${ }^{46}$ ("Le gazouillis matinal des petits oiseaux leur revenait à l'esprit et ils ne se rappelaient plus où ils étaient ni pourquoi ils étaient arrivés là »). Le bruit d'un avion militaire allemand rompt la paix et les fugitifs doivent se cacher dans la nature ; la menace extérieure se rappelle à eux. L'importance de la nature est cependant évidente dès le début qui déploie une description poétique de trois pages de la beauté de la vallée et des montagnes. L'auteur décrit avec enthousiasme et minutieusement les animaux, les plantes, le fjord et la rivière. Les tournures bibliques rappellent l'appartenance réciproque des hommes et de la nature qui ne doit pas être rompue : "Tämä oli heidän luvattuu maata " ${ }^{47}$ ("C'était leur terre promise "). On laisse comprendre également que la nature veillera sur l'homme. Le fleuve apporte une branche et la dépose sur le bord "kaffinkeittopuuksi jolleki nälkäiselle mettänkulkkijalle

44 Ibid., p. 138-139.

45 Anthony Giddens, The Consequences of Modernity, Cambridge, Polity Press, 1990, p. 114-117.

46 Claudia Deetjen, Re-imagining nature's nation, op. cit., p. 11.

47 Ibid., p. 12. 
ja lohenpyyttäjälle ${ }^{48}$ (" pour qu'un voyageur affamé ou un pêcheur de saumon puisse s'en servir pour faire du feu pour le café »). Même un tronc d'arbre pourri s'avère utile en servant de siège pour le passant. Le narrateur constate que «luonto itte pubdisteli itteensä. Samala se tuli avuksi ihmiselle " ${ }^{49}$ (" la nature se nettoya d'elle-même. En même temps elle aida l'homme»).

\section{LE RETOUR QUI RÉPARE}

Dans la troisième partie, Rauha, la guerre est finie et les gens peuvent retourner dans leurs villages détruits. Comme une partie des femmes et des enfants a été évacuée en Norvège du Sud, on pose la question de savoir s'ils devraient y demeurer pour le moment. Les villageois restés dans le Nord habitent toujours dans des kammi et la nourriture manque. Koti, le chez-soi, est cependant beaucoup plus qu'un logement. Le mot reçoit des significations presque métaphysiques. Iisakki, un des personnages principaux dans les deux premiers volumes, décrit avec lyrisme la signification profonde du chez-soi :

Täälä [Etelä-Norjassa] se ei ole lapsirukat meizän koti. Se on korkkeela pohjaisessa, missä kesänaikana aurinko paistelee yötä päivää, missä joki laulelee ikuista lauluunsa, missä tutut tunturit suojelevat meitä, missä jänkät kevväilä on valkeela hillanlehtee, ja hillanaikana loistelevat lämpimänkeltaisina hillankokkoojan silmhiin, ja missä talven yö on pitkä ja kylmä ja pimmee. Sielä se on meidän koti. ${ }^{50}$

Mes pauvres enfants, notre chez-nous n'est pas ici en Norvège du Sud. Il est dans le Nord où le soleil brille la nuit et le jour en été, où le fleuve chante sa chanson éternelle, où les montagnes nous protègent, où le jänk $\ddot{a}^{51}$ printanier est blanc de feuilles de plaquebière qui font scintiller ses baies de leur lumière jaune chaleureuse aux yeux du cueilleur en automne, où la nuit d'hiver est longue et noire. Là, nous sommes chez nous.

Limportance philosophique du chez-soi et de la nature dépasse même la religion. Arrivé au seuil de l'âge adulte, le jeune Arne trouve les règles et les prescriptions de la religion trop méticuleuses. Il n'accepte pas que la danse, la musique et les décorations de Noël soient proscrites comme des pêchés, alors qu'on oublie en même temps la guerre et la bombe atomique. Ces réflexions le conduisent à penser que la dimension spirituelle de l'homme ne peut se développer que dans la nature. Il trouve un appui à ses méditations dans la littérature classique qui célèbre la beauté et la grandeur de la nature. La poésie lyrique de Henrik Wergeland remplace la Bible dans l'esprit du jeune homme et renforce sa philosophie de la nature ${ }^{52}$.

48 Ibid., p. 7.

49 Ibid., p. 8.

50 Alf Nilsen-Børsskog, Elämän jatko 3. Rauha, Indre Billefjord, Idut, 2011, p. 411.

51 Marais typique de la région; note du traducteur.

52 Alf Nilsen-Børsskog, Elämän jatko 3, op. cit., p. 411. 
Dans la dernière partie, Viimi vuodet, Arne retourne dans la maison de son enfance où sa mère, devenue veuve, habite toujours. L'ambiance est mélancolique ; la mère et le fils se souviennent des années écoulées et des voisins et membres de la famille morts. La foi en un avenir revient cependant grâce à l'expérience de la nature. Le village a été reconstruit après la guerre, mais la nature offre un sentiment de sécurité et de continuité : "mies tunsi ittensä ollevan kotona. Rakhaala kotitörmälä!»53 («l'homme se sentit chez soi. Sur sa colline bien-aimée! »)

Les motifs du départ et du retour se répètent à travers toute la série. D’abord, un village entier part en fuite, puis le personnage principal Arne à plusieurs reprises, pour étudier, chercher du travail et construire son identité. Il fait face à la violence, la soif de pouvoir et l'exploitation brutale de l'homme et de la nature. Il retourne toujours chez lui et s'installe finalement dans sa maison natale : "Synnyintörmältänsä mies löydästi onne asuinpaikan. [...] Maailmalta onnee ei ollu löytyny $~^{54}$ ( L'homme trouva le foyer du bonheur sur sa colline natale. [...] Il ne l'avait pas trouvé dans le grand monde »). L'austérité de l'Arctique et l'immensité du désert inatteignable se répètent dans les descriptions de la nature.

Kuuntelijan sielule tunturitten rauha teki hyvvää. Pitkäksiki aikkaa mies jäi seisoskelehan kammin ulkopuolele kuuntelehan tunturimaan leppyyttellevää hiljaisuutta. [...] Oikheen hän nautiskeli päässiäisaamun säästä, joka kylvästi hänheen syvän ilon ja taivhaalisen hyvinvoinnintuntheen. Pahat ajatukset se ajasti pois ja levhäätti hänheen mitä syvviimen onnen. [...] [K] eskelä suurta sielunrauhaa mies jäiki ihhailehan tätä mahtavan kaunista luonttoo, jonka sylissä ihminen ellää, josta hän on pienen pieni osa. ${ }^{55}$

La paix de la montagne apaisait l'âme de l'homme. Il est resté longtemps en dehors du kammi pour écouter le silence réconfortant des collines. [...] Il se réjouissait du matin de Pâques qui remplissait son esprit d'une joie profonde et d'un sentiment de bien-être céleste. L'air matinal chassait les pensées noires et répandait un bonheur profond. [...] [E]ntouré de cette grande paix de l'âme Arne admirait la belle et immense nature où l'homme vit et dont il est un morceau minuscule.

La protection de la nature contre l'exploitation et l'indifférence de l'homme est un thème central, surtout dans le dernier volume de la série. La conclusion est triste : dans les derniers jours de sa vie, le vieux Arne écoute les informations à la radio et constate que l'homme n'a rien appris : «omasta verisestä historiastakhaan. [...] Ihminen ryözyttää meret, maat ja ilmatki " ${ }^{56}$ (" de sa propre histoire sanglante. [...] L'homme pollue la mer, la terre, et même l'air »).

53 Alf Nilsen-Børsskog, Elämän jatko 4. Viimi vuodet, Indre Billefjord, Idut, 2015, p. 9.

54 Ibid., p. 488.

55 Ibid., p. 482.

56 Ibid., p. 501. 
Dans ses romans, Alf Nilsen-Børsskog rend visible un récit sur l'histoire proche de la Norvège qui n'a pas été raconté auparavant. La tactique de la terre brûlée de l'armée nazie a détruit presque complètement toute la propriété des habitants des villages kvènes de la côte de la mer Arctique ; leur patrimoine culturel a failli disparaître entièrement. Après la guerre, il était difficile sinon impossible de construire l'identité sur les anciennes fondations. La petite minorité ethnique s'est trouvée à la merci de la société dominante.

Les romans de Nilsen-Børsskog décrivent une identité traditionnelle fondée sur un mode de vie collective ainsi que les possibilités qui s'ouvrent à la construction identitaire après une rupture subite et brutale. Les ingrédients d'un sentiment de sécurité spirituelle se trouvent dans la nature et l'environnement. La nature arctique de la région d'origine représente la continuité et l'équilibre. Les paysages et l'environnement sont dépositaires des significations et du symbolisme qui rattachent l'homme à son histoire personnelle et à la chaîne des générations.

Traduit par Harri Veivo 